\title{
The Protection and Inheritance of Dunhuang Culture in Intercultural Communication
}

\author{
Xi Zhang \\ School of Art and Design, Lanzhou Jiaotong University, Lanzhou 730070, China. \\ 59564008@qq.com
}

\begin{abstract}
Dunhuang culture faces the problem of cross cultural communication with the limitation of communication, the difference of communication, and the transmission of aphasia. In practice, the tendency of the marginalization of Dunhuang an.d the industrialization of the Dunhuang culture is appeared. It is need to protect and develop Dunhuang culture in cross culture communication through the key protection, retention of native, digital protection strategy and cultivate qualified "gatekeeper", cultivate qualified international audience, expand a wide range of "communication channels" strategy.
\end{abstract}

Keywords: Dunhuang culture; intercultural communication; protection and inheritance.

\section{Introduction}

Entering the 21st century, the global pattern shows a "center - periphery" of the situation: developed countries and regions are the central source of communication. They not only occupy a prime location for cultural dissemination and transfer to the developing countries a set of ideological systems and value concepts. The spread of information in developed countries and developed areas has more than $80 \%$ of the total world information flow, the United States accounted for more than $60 \%$ [1].

\section{Present Situation of Dunhuang culture in Intercultural Communication}

\subsection{The Limitation of the Cultural Transmission.}

The culture of Dunhuang has obvious regional characteristics, which is very rich in natural tension and cultural connotation, which is obviously different from the culture of other nations in the world. Regional Dunhuang culture has gained a strong sense of benign development initiative which can rely on a variety of modern communication methods and approaches far beyond the original domain limit, to the wider world. Edward Said, said: "Every culture needs the development and maintenance of a heterogeneous there to and the other self-existence with its competing involves the construction of self-identity and its own opposite 'otherness' identity. Construction and always involved and continue to explain 'us' different characteristics and re-interpretation. Each age and society re-create their own 'otherness'. Therefore, self-identity or 'otherness' identity is by no means static stuff. As "other" culture, Dunhuang culture is a kind of" relationship exists". It is suitable to explore and utilize the resources effectively in the communication and interaction, and it can be used in many aspects.

\subsection{The Difference of Cultural Communication.}

Cultural diversity is to preach the spread of cultural areas by both sides, time, religion has led to cultural differences in understanding. In terms of historical occurrence, Various nature and form of cultural differences rooted in a variety of special ethnic, linguistic, geographical and natural conditions [2]. Cultural differences can lead the audience held cultural standpoint with steady, dominant and exclusive. The typical differences of cultural communication in Dunhuang lay in:

The difference of Cultural. Cultural of Different regional has huge cultural quest. The history of region cultural is difficult to find common ground be understood and spread to audiences who has been have long experience for of different cultural heritage. 
The difference of time. The Dunhuang culture began form early of the Qin dynasty, and it has a long history. With constantly changing dynasties, it has gradually lost its cultural connotation semantics.

The difference of religious. Dunhuang culture is a regional culture that based on the Buddhism developed, whereas audience of intercultural communication don't understand Buddhism all of them. External cultural system of religious culture in diverse forms, and it including Christianity, Islam and so on.

Modern Western culture consciousness to a certain extent is a kind of cultural differences consciousness. Western culture from maintaining their cultural beliefs and cultural status of departure, it have ensure that mainstream Western culture and to remove his cultural snatch of the cultural status, so fully aware of the differences between Dunhuang culture and western culture. Dunhuang culture shaping their own way of mainstream culture, no more than three kinds: One is to build a unified culture of this ideology, suppress or exclude other regional culture and implementation of the cultural tyranny. Dunhuang culture obviously do not have this kind of cultural transmission strength. The second way is through negotiation of Western culture and the Dunhuang culture of negotiation to reach a certain degree of "balanced compromise", then maintain and develop the Dunhuang culture in the diverse cultural environment. A third way is make Dunhuang culture as the main body to actively absorb the beneficial elements of modern Western culture and enrich the connotation of Dunhuang culture, the growth of Dunhuang cultural vitality.

\subsection{The Aphasia of Culture Communication.}

Many linguists have compiled some books that fully affirm the necessity and importance in the understanding of the object of culture in intercultural communication and foreign language learning process, but it ignores the cultural exchange is bidirectional basic theory and despise their culture Education and output that leads to their cultural aphasia [3]. Due to the resistance of the overall content of the traditional culture and Western culture unique, Dunhuang culture inevitably encounter cultural barriers in information transferring.

In Intercultural Communication, Dunhuang culture Aphasia mainly point in two aspects: Chinese people understand Dunhuang local culture cannot use correct English expression when they take in intercultural communication. This results in communication failure or inefficient. Foreigners do not understand or even misunderstand Chinese culture. To overcome this deficiency, we should start from the English training. The research on exotic cultural differences has been an urgent need to avoid an obstacle in the process of cross-cultural communication.

\section{The Trends of Dunhuang Culture in Intercultural communication}

\subsection{The Marginalization of Dunhuang Ontology.}

In this diverse pattern of interests, as a symbol of local culture, Dunhuang disappeared with the disappearance of the historical and cultural ecology. Its meaning and form begins to be stripped. Industrialization leads to Dunhuang cultural lifestyle away from public life, only to be regarded as a cultural symbol with aesthetic rather than functionality.

Over the years, the spread of Dunhuang culture has been relying on Dunhuang tourism and the support of government policy, Dunhuang culture weakens or abandons the connotation of the traditional charm and Buddhist seeks gorgeous, color and shape public aesthetic under the dilemma faced by marginalized. A number of profound Dunhuang culture 'kept in purdah with no one knowing', divorced from the edge of the world's cultural development.

\subsection{Industrialization of Dunhuang Derived Culture.}

Based on the Dunhuang cultural, the new Dunhuang artistic products faced the general public. The most representative work is dance drama "Silk Road" which has showed for thirty years. Then, many dance dramas have been out, such as "Dream of Dunhuang", "Dunhuang", and "Dunhuang goddess In addition, there are Dunhuang arts and crafts, Dunhuang music and dance, Dunhuang Literature, Dunhuang movie and TV drama, Dunhuang print publications, Dunhuang painting and so on. In the 
audience experience of a larger market, the economic pleasure of industrialization directly challenged the evolution of local culture.

The development of Dunhuang cultural resources directly concerns the cultural regeneration capacity in the context of globalization. In time and space which would not been foreseen, it is possible to accelerate the trend of national cultural heterogeneity and abstraction. This cultural anxiety causes ecological imbalance which will not only create obstacles to national cultural awareness, but also cause mental distress cultural reconstruction. Because cultural development is not only the performance of the evolution of ritual or symbolic, it also reflects in the internal driving force of sustainable development Contact [4].

\section{The Protection and Inheritance Strategy in Intercultural Communication}

\subsection{Protection Strategy.}

Determining key points for conservation. Culture is a reflection of the spirit of era, advancing with the ages. Cultural development has a strong inclusive that often shows a coexistence of essence and dross. Dunhuang cultural history involves long, wide area, complicated forms. The content is relatively complex. It can only take methods to point for its development and protection. The individual highlight culture such as painted sculptures, murals, should be fully developed, making it to be the country and the world people favorite items.

Retaining the original ecological culture. Dunhuang culture belongs to the category of social history and culture. Its history and culture with the other history and culture are reflected in real-life history. Dunhuang culture reflects the region's production and life of local people, myths and religious beliefs. For the development and protection of the cultural heritage should retain its traditional antique culture. Appropriate transformation of their feudal superstitious basic also owns the original culture. Inheriting excellent national culture is on the basis of traditional sports. Comrade Deng Xiaoping said that there should be two aspects of arts and culture in the form of development, one is more complex to develop advanced form, and on the other hand it should be relatively simple to popular forms of development. Use the old form to reflect new content approach is also necessary.

Digital technology protection of Dunhuang culture. Firstly, in the longitudinal, digitized explore historical and cultural relics should be able to protect and support the role of inheritance lasted; secondly, in the transverse direction, digital means should contribute to promoting the history of civilization in current. The essence of traditional culture conducive contributes to the propagation properties.

\subsection{Development Strategy.}

To train qualified "gatekeeper". In terms of dissemination of the body, Dunhuang culture spread to train qualified "gatekeeper" - proficient in Chinese and Western cross-cultural communicator. The context of globalization, the spread of Dunhuang culture planners should have international vision, perspective and overweight abandon single native complex, with modern, globalized vision to take care of Dunhuang culture. Dunhuang cultural transmission planner specializing in Dunhuang culture refers to external communication planners. They are the cultural, artistic communication messenger, but also the spread of Dunhuang culture "gatekeeper." "Gatekeeper" role Dunhuang culture determines the overall quality of the external communicators.

To train qualified "international audience". In terms of the spread of the object, "an international audience" is important .Dunhuang culture handles "set the agenda", uses the correct cultural communication strategy so that foreign audiences can understand and love Chinese culture. Through contacting Dunhuang culture to culture keen interest, Through living environment and cultural atmosphere of subtle to prompte cultural identity."Because cultural development is the integration of foreign and local culture to learn from each other and realize through cultural development which is a dynamic process. Pluralistic contested synthetic culture undeniably remains local which continue to evolve and become new starting point "[5]. 
Expand a wide range of communication channels. Dunhuang's cultural industry is supported by rich traditional cultural heritage. The content of its industry is not only limited to the tourism industry, but should include other creative expression. Such as theatrical performances, film and video, literature, painting and calligraphy. Let the cultural products use "commodity circulation" wide channel, realize the circulation of the Dunhuang culture in globalization.

\section{Conclusion}

Dunhuang culture faces the problem of cross cultural communication with the limitation of communication, the difference of communication, and the transmission of aphasia while the global pattern shows a "center - periphery" of the situation. In practice, the tendency of the marginalization of Dunhuang and the industrialization of the Dunhuang culture is appeared. It is need to protect and develope Dunhuang culture in cross culture communication through the key protection, retention of native, digital protection strategy and cultivate qualified "gatekeeper", cultivate qualified international audience, expand a wide range of "communication channels" strategy.

\section{Acknowledgements}

Thanks for National Arts Fund 2013 the protection and Heritage of Dunhuang sculpture art.

\section{References}

[1] Yin Hong, Li Bin. Globalization and mass media. Tsinghua University Press, 2002, P. 199.

[2] Ouyang Kang. Cross-cultural understanding and communication. Social science, Vol. 6 (1997).

[3] Zhang LAN, Intercultural Communication of Chinese Culture Aphasia analysis .Southwest Nationalities University (Social Sciences), Vol. 8 (2003), p. 339-341.

[4] Wang Jing, Researches on the Intercultural Communication Strategy of Chinese martial arts cultural identity. Chinese school sports, Vol. 8(2014), p. 10-15.

[5] Chen taowen. Not open is not enough to analyze cultural globalization in media protection and media open [G] // Yin Hong, Li Bin globalization and mass media: Conflict, Fusion - Interactive. Tsinghua University Press, 2002, p. 324. 